\title{
Liste des thèses d'anthropologie soutenues en 2012
}

\section{Université d'Aix-Marseille}

Emilie BUTTARELLI : Croissance et alimentation $d u$ jeune enfant (0-3 ans) en milieu urbain à Dakar (Sénégal)

Emmanuel COHEN : L'image du corps chez les Sénégalais : applications à l'étude de l'obésité dans un contexte de transition nutritionnelle

Philippe STEFANINI : La spiruline : essai anthropologique sur le processus de conversion éthique des agriculteurs varois en quête de sens

\section{Université Bordeaux-I}

Géraldine SACHAU-CARCEL : Apport de la modélisation tridimensionnelle à la compréhension du fonctionnement des sépultures multiples. L'exemple du secteur central de la catacombe des Saints-Pierre-et-Marcellin (Rome, Italie) [I ${ }^{\text {er}}$-milieu III ${ }^{e}$ siècles apr. J.-C.]

Emmanuel D'INCAU : Hypercémentose: définition, classification et fréquence. Apport des résultats à la lignée néandertalienne

\section{Université de Toulouse-III}

Rémi ESCLASSAN : Étude des caries en fonction $d u$ sexe au sein d'individus adultes de la population médiévale (IX ${ }^{e}-X V^{e}$ siècles) de Vilarnau (Pyrénées-Orientales et synthèse sur l'usure)

\section{Muséum national d'histoire naturelle}

Noémie BECKER: Facteurs génétiques et sélection naturelle impliqués dans la faible stature des populations pygmées d'Afrique centrale

Émeric BILLARD : Nouveaux acteurs, vieilles habitudes. L'implantation des opérateurs forestiers asiatiques au Gabon à l'heure de la transition vers la gestion durable

Noémie BONNEAU : Analyse tridimensionnelle du complexe pelvi-fémoral : son fonctionnement et sa mise en place au cours de l'ontogenèse. Dimension évolutive et applications cliniques

Elodie REGHEM : Stratégies comportementales et cinématiques de la préhension des primates 